\title{
Tremor in motor neuron disease may be central rather than peripheral in origin
}

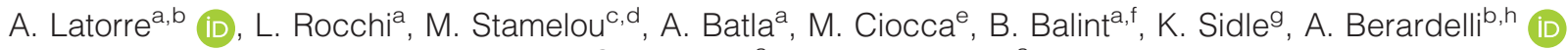 \\ J. C. Rothwell and K. P. Bhatia ${ }^{a}$
}

\begin{abstract}
${ }^{a}$ Sobell Department of Motor Neuroscience and Movement Disorders, University College London Institute of Neurology, London, UK;
${ }^{\mathrm{b}}$ Department of Neurology and Psychiatry, Sapienza, University of Rome, Rome, Italy; ${ }^{\mathrm{c}}$ Department of Neurology, Philipps University, Marburg, Germany; 'Parkinson's Disease and Movement Disorders Department, HYGEIA Hospital, Athens, Greece; 'Department of Neurology, Fatebenefratelli Hospital, ASST Fatebenefratelli Sacco, Milan, Italy; ${ }^{\mathrm{f}}$ Department of Neurology, University Hospital Heidelberg, Heidelberg, Germany; ${ }^{\mathrm{g}}$ Department of Clinical Neuroscience, University College London Institute of Neurology, London, UK; and ${ }^{\mathrm{h}}$ Istituto di Ricovero e Cura a Carattere Scientifico (IRCCS) Neuromed, Pozzilli, Italy
\end{abstract}

\section{Keywords:}

cerebellum, eyeblink classic conditioning, motor neuron disease, power spectrum, tremor

Received 12 January 2018 Accepted 21 June 2018

European Journal of Neurology 2018, 0: 1-7 doi:10.1111/ene.13743
Background and purpose: Motor neuron disease (MND) refers to a spectrum of degenerative diseases affecting motor neurons. Recent clinical and postmortem observations have revealed considerable variability in the phenotype. Rhythmic involuntary oscillations of the hands during action, resembling tremor, can occur in MND, but their pathophysiology has not yet been investigated.

Methods: A total of 120 consecutive patients with MND were screened for tremor. Twelve patients with action tremor and no other movement disorders were found. Ten took part in the study. Tremor was recorded bilaterally using surface electromyography (EMG) and triaxial accelerometer, with and without a variable weight load. Power spectra of rectified EMG and accelerometric signal were calculated. To investigate a possible cerebellar involvement, eyeblink classic conditioning was performed in five patients.

Results: Action tremor was present in about $10 \%$ of our population. All patients showed distal postural tremor of low amplitude and constant frequency, bilateral with a small degree of asymmetry. Two also showed simple kinetic tremor. A peak at the EMG and accelerometric recordings ranging from 4 to $12 \mathrm{~Hz}$ was found in all patients. Loading did not change peak frequency in either the electromyographic or accelerometric power spectra. Compared with healthy volunteers, patients had a smaller number of conditioned responses during eyeblink classic conditioning.

Conclusions: Our data suggest that patients with MND can present with action tremor of a central origin, possibly due to a cerebellar dysfunction. This evidence supports the novel idea of MND as a multisystem neurodegenerative disease and that action tremor can be part of this condition.

\section{Introduction}

Motor neuron disease (MND) refers to a spectrum of progressive degenerative diseases that variably affect the upper and lower motor neurons, and includes a continuum that spans from primary lateral sclerosis to

Correspondence: K. P. Bhatia, University College London, 33 Queen Square, London WC1N 3BG, UK (tel.: 44203448 8723; fax: 44203448 3954; e-mail: k.bhatia@ucl.ac.uk). progressive muscular atrophy. Amyotrophic lateral sclerosis (ALS) is the most common subtype of MND and is characterized by predominant lower motor neuron signs combined with a degree of pyramidal signs due to upper motor neuron involvement [1]. Clinical and post-mortem observations demonstrated that other neural structures can be involved, indicating that MND should be regarded as a widespread degenerative disease of the central nervous system (CNS) [2-4]. 
In 1970, Spiro used the term minipolymyoclonus to clinically describe intermittent and irregular movements, with amplitudes just sufficient to produce visible and palpable movements of the joints' observed in childhood spinal muscular atrophy [5]; these were then attributed to fasciculations [6]. However, in our clinical experience, patients with MND often show rhythmic involuntary oscillations of the hands during action, with a fairly constant frequency and not associated with fasciculations, thus resembling tremor.

Tremor recognizes different pathophysiological mechanisms, such as mechanical resonance, enhanced short- or long-latency reflexes and central oscillations caused by a network dysfunction [7]. Mechanicalreflex tremor is influenced by the inertial and elastic properties of the body; consequently, inertial loads on a joint decrease its frequency [8]. By contrast, central tremors are not affected by mechanical changes, as they are produced by a rhythmic activity generated within the CNS [9].

Tremor in MND might have diverse origins. One possibility is that it is due to peripheral factors, such as weakness caused by denervation or spasticity resulting from upper motor neuron damage. However, given the diffuse damage to the CNS during the course of the disease, a central origin cannot be ruled out. The cerebellum and olivocerebellar network are presumed to drive high-frequency $(6-40 \mathrm{~Hz})$ oscillations of the neocortex and probably play a critical role in pacing several types of tremors [7,9-11]. Dysfunctions of the olivocerebellar network can be investigated through an associative learning paradigm known as eyeblink classical conditioning (EBCC) [12].

In this study, we sought to characterize the clinical and neurophysiological features of the observed action tremor in MND. To do so, we confirmed the tremulous nature of the involuntary movements through power spectrum analysis and measured possible frequency changes due to inertial loading. Moreover, to explore a possible involvement of the cerebellum, we performed EBCC in a subgroup of tremulous patients with MND and compared the results with healthy volunteers.

\section{Methods}

\section{Participants}

A total of 120 consecutive patients with MND attending the MND outpatients' clinic at the National Hospital for Neurology and Neurosurgery (London, UK) were examined for tremor over a 12-month period. Twelve patients with action tremor and no other movement disorders were found and 10 of them were recruited. Parkinsonism and other secondary causes of action tremor were excluded. Participants gave their written informed consent. All experimental procedures were approved by the University College London Research Ethics Committee, and were conducted in agreement with the Declaration of Helsinki and according to international safety guidelines.

\section{Clinical examination}

Information about demographic and clinical data was collected, including age at onset, disease duration, tremor duration, handedness, weakest body side and body side most affected by tremor (Table 1). Muscle strength was assessed clinically in the flexor carpi radialis (FCR) and extensor carpi radialis (ECR) muscles bilaterally using the Medical Research Council (MRC) power scale. Grip strength was measured by a hand dynamometer ranging from 0 to $90 \mathrm{~kg}$ (JAMAR ${ }^{\circledR}$ hydraulic, S.I. Instruments, Hilton, SA, Australia). Tremor was evaluated at rest and during posture (arms outstretched) and action (finger-to-nose test, when possible).

\section{Tremor recording}

Tremor was recorded bilaterally from the FCR and ECR through surface electromyography (EMG) using bipolar $\mathrm{Ag}-\mathrm{AgCl}$ cup electrodes. The continuous EMG signal was bandpass filtered $(3-100 \mathrm{~Hz})$, rectified and linearly detrended prior to further analysis. A fast Fourier transform using non-overlapping segments of $2^{14}$ points was used for frequency decomposition. Autospectra were measured using functions

Table 1 Patients' demographic and clinical data

\begin{tabular}{lllllll}
\hline Patient & $\begin{array}{l}\text { Age } \\
\text { (years) }\end{array}$ & Gender & Diagnosis & $\begin{array}{l}\text { Site of } \\
\text { onset }\end{array}$ & $\begin{array}{l}\text { DD } \\
\text { (years) }\end{array}$ & $\begin{array}{l}\text { TD } \\
\text { (years) }\end{array}$ \\
\hline 1 & 78 & M & ALS & Right hand & 6 & 1 \\
2 & 61 & M & PMA & Upper limbs & 6 & 1 \\
3 & 43 & M & ALS & Left hand & 7 & 1 \\
4 & 46 & M & ALS & Left hand & 10 & 8 \\
5 & 74 & M & ALS & Left hand & 4 & 0.3 \\
6 & 55 & M & ALS & Left foot & 9 & 3 \\
& & & (SOD1) & & & \\
7 & 68 & M & PBP & Bulbar & 6 & 3 \\
8 & 63 & M & ALS & Left hand & 3 & 2.5 \\
9 & 72 & M & ALS & Bulbar & 2 & 1 \\
10 & 76 & M & ALS & Upper & 7 & 0.5 \\
& & & & limbs & & \\
Average & 64 & & & & 6 & 2.1 \\
SD & 12.4 & & & & 2.5 & 2.3 \\
\hline
\end{tabular}

ALS, amyotrophic lateral sclerosis; DD, disease duration at tremor registration; M, male; PBP, progressive bulbar palsy; PMA, progressive muscular atrophy; SOD1, mutations in superoxide dismutase 1; TD, tremor duration. 
contained in the Neurospec toolbox $[13,14]$ written for MATLAB software (Mathworks, Natick, MA, USA). Tremor was also recorded by a triaxial accelerometer (ACC) placed on the dorsal surface of the most affected hand while subjects were seated with arms outstretched at shoulder level. These signals were collected through a CED 1401 interface (Cambridge Electronic Design, Cambridge, UK), digitized at a sampling rate of $5 \mathrm{kHz}$ and fed to a computer for data storage using Spike2 software (Cambridge Electronic Design). Tremor was recorded for at least $60 \mathrm{~s}$ in two loading conditions: without weight ('no weight') and with a variable weight load (100, 200 or $500 \mathrm{~g}$ ) based on patients' muscle power ('weight') located on the metacarpal region of the hands.

\section{Eyeblink classic conditioning}

Eyeblink classic conditioning was performed in a subgroup of five patients according to a previously described protocol [15]. The conditioning stimulus (CS) was a tone lasting $400 \mathrm{~ms}$, delivered via binaural headphones and loud enough (70-80 dB; $2 \mathrm{kHz}$ ) to inconsistently produce an 'alpha blink' within $200 \mathrm{~ms}$ [15]. The unconditioned stimulus (US) was a square electrical pulse of $200-\mu$ s duration and an intensity equal to twice that required to obtain a motor response in the orbicularis oculi of $50-100 \mu \mathrm{V}$, delivered over the right supraorbital nerve $400 \mathrm{~ms}$ after the CS. Pairs of CS and US at an interstimulus interval of $400 \mathrm{~ms}$ were delivered in six acquisition blocks. Each block consisted of nine CS-US pairs, one US-only and one CS-only trial; a seventh block consisted of 11 CS-only trials to measure extinction. Surface EMG was recorded bilaterally from the orbicularis oculi muscle. All of the EMG bursts occurring at least $200 \mathrm{~ms}$ after the CS but before the US were considered as conditioned responses (CRs) in CS-US trials. For CSonly trials, EMG bursts occurring 200-600 ms after the CS were considered CRs. Patient results were compared with those obtained from 12 healthy subjects.

\section{Statistical analysis}

Several $t$-tests were used to compare differences in peak frequency (PF) recorded by EMG and ACC to investigate possible effects of load on peak tremor frequency and to compare grip strength between the right and left side. Wilcoxon's signed-rank test was used to disclose possible differences in muscle strength across the four examined muscles and Mann-Whitney tests were performed to compare the number of conditioned EBCC responses in each block in the two groups (patients with MND and healthy subjects). Spearman's correlation coefficient was used to investigate a possible correlation between neurophysiological and clinical variables. When using $t$-tests, normal distribution of data was assessed by means of a Shapiro-Wilks test. $P<0.05$ was considered significant.

\section{Results}

Action tremor was present in about $10 \%$ of our MND population. All of the recruited patients presented with postural tremor and two of them presented with both postural and kinetic tremor. Rest and intention tremor were not observed. Eight of the patients had a diagnosis of ALS, whereas the other two fitted the criteria of progressive muscular atrophy and progressive bulbar palsy, respectively. Clinical features of patients who took part in the study are summarized in Tables 1 and 2.

\section{Tremor recording}

All patients showed a consistent peak in the EMG and ACC recording (Figs 1 and 2). EMG PF ranged from 4.0 to $9.5 \mathrm{~Hz}$ (average $7.2 \pm 2.1 \mathrm{~Hz}$ ) and from 4.0 to $10.4 \mathrm{~Hz}$ (average $7.5 \pm 2.4 \mathrm{~Hz}$ ) in the "no weight' and 'weight' condition, respectively. ACC PF varied from 4.3 to $12.2 \mathrm{~Hz}$ in the 'no weight' condition (average $7.3 \pm 2.4 \mathrm{~Hz}$ ) and from 4.6 to $12.8 \mathrm{~Hz}$ in the 'weight' condition (average $7.3 \pm 2.6 \mathrm{~Hz}$ ). $t$-tests did not show any significant differences in PF between EMG and ACC in either the 'no weight' ( $\left.\mathrm{t}_{9}=0.371, \quad P=0.720\right) \quad$ or $\quad$ 'weight' $\quad\left(\mathrm{t}_{9}=-0.434\right.$, $P=0.674)$ condition. Loading did not change PF measured with either EMG $\left(\mathrm{t}_{9}=-1.682, P=0.127\right)$ or ACC $\left(\mathrm{t}_{9}=0.318, P=0.758\right)$.

There was no difference in average grip strength between the right and left side in patients with MND ( $\mathrm{t}_{9}=0.745, P=0.475$ ) and Wilcoxon's signed rank test did not disclose any significant differences in strength across the four muscles examined (all $P$-values $>0.05$ ).

Compared with healthy volunteers, patients with MND had fewer CRs during EBCC in blocks 3 $(Z=-2.472, P=0.014), 4(Z=-2.638, P=0.007), 5$ $(Z=-2.830, \quad P=0.004)$ and $6(Z=-2.680, \quad P=$ 0.004) (Fig. 3).

There was a positive correlation (Spearman's rank) between tremor duration and the presence of kinetic tremor $(r=0.676, P=0.032)$. No correlation was found between the degree of atrophy in the FCR or ECR muscles and tremor power measured in the ACC signal. 
Table 2 Main clinical variables

\begin{tabular}{|c|c|c|c|c|c|c|c|c|c|c|c|}
\hline \multicolumn{5}{|c|}{ Medical Research Council (MRC) power scale } & \multicolumn{5}{|c|}{ Grip strength (kg) } & \multirow[b]{2}{*}{ Atrophy } & \multirow{2}{*}{$\begin{array}{l}\text { Kinetic } \\
\text { tremor }\end{array}$} \\
\hline Patient & FCR-D & ECR-D & FCR-ND & ECR-ND & $\mathrm{D}$ & $\mathrm{ND}$ & Load $(\mathrm{g})$ & WS & TS & & \\
\hline 1 & 2 & 2 & 3 & 3 & 2 & 7 & 500 & $\mathrm{R}$ & $\mathrm{R}$ & Severe & - \\
\hline 2 & 4 & 4 & 4 & 4 & 14.5 & 11 & 500 & $\mathrm{~L}$ & $\mathrm{R}$ & Mild & - \\
\hline 3 & 5 & 4 & 5 & 5 & NA & NA & 500 & $=$ & $\mathrm{L}$ & Absent & - \\
\hline 4 & 2 & 2 & 2 & 2 & 4 & 2 & 100 & $\mathrm{~L}$ & $\mathrm{R}$ & Severe & + \\
\hline 5 & 4 & 5 & 4 & 4 & NA & NA & 500 & $\mathrm{R}$ & $\mathrm{R}$ & Mild & - \\
\hline 6 & 1 & 0 & 1 & 0 & 0 & 0 & 200 & $=$ & $\mathrm{R}$ & Severe & - \\
\hline 7 & 5 & 5 & 5 & 5 & 11 & 12 & 500 & $\mathrm{R}$ & $\mathrm{L}$ & Absent & + \\
\hline 8 & 3 & 2 & 2 & 2 & 1 & 0 & 200 & $\mathrm{~L}$ & $\mathrm{~L}$ & Severe & - \\
\hline 9 & 2 & 2 & 3 & 3 & 1 & 10 & 500 & $\mathrm{R}$ & $\mathrm{L}$ & Severe & - \\
\hline 10 & 3 & 3 & 3 & 3 & 2 & 2 & 100 & $=$ & $\mathrm{L}$ & Severe & - \\
\hline Average & & & & & 4.4 & 5.5 & & & & & \\
\hline SD & & & & & 5.3 & 5.1 & & & & & \\
\hline
\end{tabular}

D, dominant; ECR, extensor carpi radialis; FCR, flexor carpi radialis; L, left; NA, not available; ND, non-dominant; R, right; TS, body side

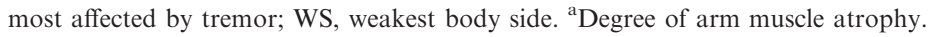

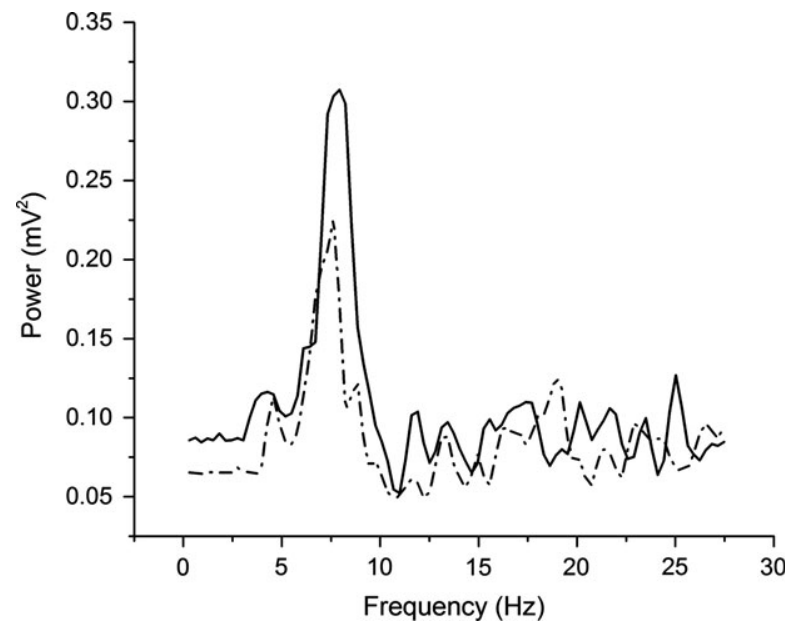

Figure 1 An example of electromyography power spectrum showing a peak at 7.6 and $7.9 \mathrm{~Hz}$ in the two loading conditions. Dashed black line, with weight; solid black line, without weight.

\section{Discussion}

We described 10 patients with MND who presented with bilateral postural tremor and no other moment disorders; two of them also showed simple kinetic tremor. Postural tremor was distal, of low amplitude and constant frequency, and bilateral with a small degree of asymmetry. In none of the patients was there a clear pattern (i.e. alternating or co-contracting) of EMG activation in FCR/ECR pairs. Spectral analysis of the EMG and ACC recording showed a consistent peak of frequency ranging from 4 to $12 \mathrm{~Hz}$, which was not affected by external loading. Lastly, patients with MND consistently showed fewer CRs during EBCC compared with healthy subjects.

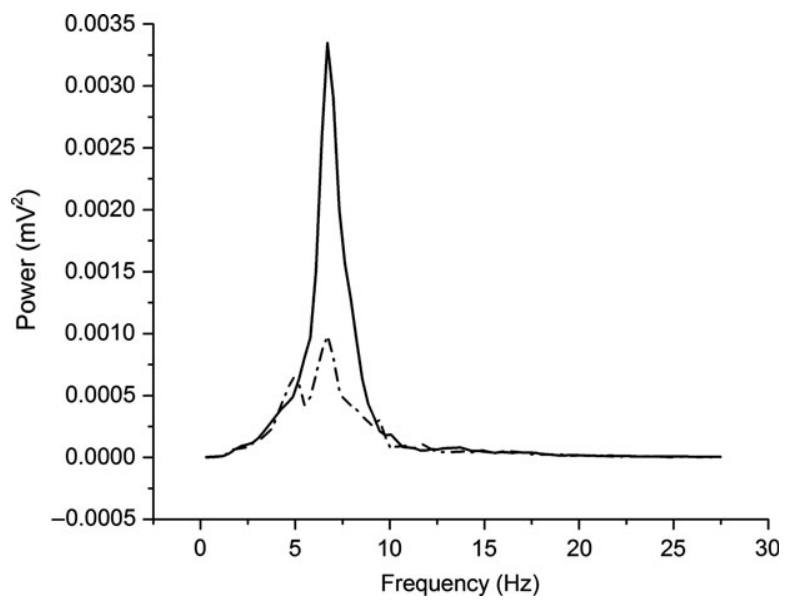

Figure 2 An example of accelerometer power spectrum showing a peak at $6.7 \mathrm{~Hz}$ in the two loading conditions. Dashed black line, with weight; solid black line, without weight.

Overall, these findings suggest that our patients with MND presented with tremor probably due to a central oscillator.

A purely mechanical tremor, such as physiological tremor, is induced by passive mechanical oscillations, the frequency of which depends on inertia and stiffness of the joint, with no clear EMG counterpart [16]. By contrast, in pathological tremors [such as enhanced physiological tremor, essential tremor (ET) and Parkinsonian tremor], motor unit entrainment is strong enough to produce a clear peak in the EMG power spectrum [16,17]. This means that the CNS provides a regular series of phasic impulses to the muscles, which might originate from unstable stretch reflex loops, central oscillators or a combination of the two [17]. The muscle stretch reflex can induce 


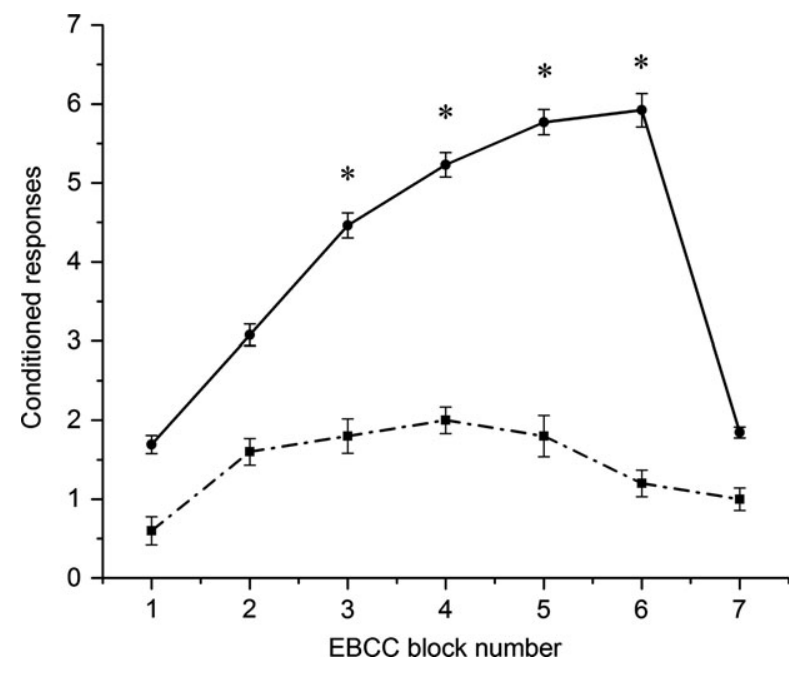

Figure 3 Eyeblink classic conditioning (EBCC) result. Conditioned responses were lower in patients $(\mathbf{\square})$ with motor neuron disease than healthy subjects (-) in blocks 3-6. Error bars indicate standard error. *Significant differences in post hoc comparisons $(P<0.05)$.

pathological tremor on its own, if its gain is increased by a relatively small amount [18], and can easily reinforce an ongoing tremor as a consequence of the inevitable delay between application of stretch and recruitment of muscle force [17]. Hence, the stretch reflex can contribute to all pathological tremors, specifically the so-called mechanical-reflex tremor [9] and central tremors, originating within the CNS, whose frequency is independent of mechanical limb properties $[17,19,20]$. Central tremor generators can be of two types: an unstable loop circuit or a neuronal ensemble with spontaneous rhythmicity that drives an otherwise normal circuit [7]. The cerebellothalamocortical network has long been suspected to be the generator of both Parkinsonian tremor and ET [7], and the spontaneously rhythmic neurons of several nuclei are the principal candidates as central tremor generators $[9,17,21]$.

In all of our patients, EMG analysis showed a clear peak in the spectrum and no frequency changes with mechanical loading, excluding fasciculations, arrhythmic involuntary movements (such as myoclonus) and mechanical or mechanical-reflex tremor. Therefore, we conclude that the tremor in MND is likely to be of central origin. We did not assess whether abnormal stretch reflex excitability influenced motor unit entrainment; thus we cannot exclude that enhanced stretch reflexes support a central loop rhythmically activating the tremulous muscle [22].

A second finding in our study is an abnormal EBCC in our patients, suggesting that the cerebellum might be a contributor to the central circuit driving tremor. EBCC is an associative learning paradigm that depends on the integrity of the olivocerebellar circuit [12] and it is known that a structural or functional impairment of the cerebellum leads to abnormalities in acquisition of CR [23]. Dysfunctions of circuits involving the cerebellum and inferior olives have been reported to play a critical role in the pathophysiology of action-induced tremors [11] and EBCC has been found to be abnormal in ET [24] and dystonic tremor $[15,23]$. Similarly, we might speculate that MND action tremor is due to a cerebellar dysfunction. Structural and functional cerebellar abnormalities in ALS have recently been demonstrated by imaging [25-27] and pathological $[28,29]$ studies, especially in patients with ALS with abnormal repeat expansions in the C9orf72 [30,31] and ataxin-2 genes (ATXN2) [27,32]. None of our patients with MND presented with cerebellar signs or were tested for intermediate ATXN2 expansions but, in one patient, C9orf72 mutation was not found and one patient carried a mutation in the SOD1 gene, whose overexpression leads to Purkinje cell degeneration in mice [33].

A possible link between tremor and ALS could be explained by the FUS gene. This gene was identified as a risk factor for both familial and sporadic ALS [34-36], and there is growing evidence that variants in the FUS gene are associated with ET [37-39]. Although the findings suggest that FUS in ALS and ET might have different pathogenetic mechanisms, this has not yet been demonstrated [40]. Therefore, possible overlapping FUS mutations in the two conditions cannot be excluded.

Our patients presented with other atypical features apart from tremor, such as a long disease duration and a slow rate of progression, not resembling the 'classical' form of ALS, which usually has a median survival of 3 years from diagnosis. Interestingly, the same characteristics, including tremor, have been described in some families with ALS type 8 caused by VAPB gene mutations [41,42]. It is thus possible to presume that those patients represent a variant of MND.

Kinetic tremor was also noticed in two of our patients [43]. None seemed to have intention tremor, but this evaluation was limited by the poor motor performance in most of them. Curiously, the presence of kinetic tremor positively correlated with disease duration, which is different from what it is seen in most of the tremors. In ET, for instance, disease duration has been correlated only to the presence of intention tremor, but it has been suggested that the presence of kinetic tremor implies a higher severity of ET [44]. Using the same analogy, we may argue that tremor in MND worsens with disease progression. 
Some limitations in our study should be acknowledged. First, patients were studied with different loading weights and these were selected according to their ability to hold the weight for $60 \mathrm{~s}$. Unfortunately, because of the severe disability and the poor general condition that this disease causes, we were not able to recruit control patients without tremor. Moreover, for the same reason, we could not assess stretch reflex excitability and denervation at the time of the tremor recording. Denervation might contribute to tremor; however, the absence of correlation between muscle atrophy and tremor power might support a central origin of tremor in MND.

In conclusion, the present study demonstrates that action tremor occurs in approximately $10 \%$ of our patients with MND and has a central origin, possibly resulting from a cerebellar dysfunction. This evidence supports the novel idea of MND as a multisystem neurodegenerative disease and suggests that action tremor is part of the condition.

\section{Disclosure of conflicts of interest}

The authors declare no financial or other conflicts of interest.

\section{References}

1. Sabatelli M, Conte A, Zollino M. Clinical and genetic heterogeneity of amyotrophic lateral sclerosis. Clin Genet 2013; 83: 408-416.

2. Lowe J. New pathological findings in amyotrophic lateral sclerosis. J Neurol Sci 1994; 124: 38-51.

3. Braak H, Brettschneider J, Ludolph AC, Lee VM, Trojanowski JQ, Del Tredici K. Amyotrophic lateral sclerosis - a model of corticofugal axonal spread. Nat Rev Neurol 2013; 9: 708-714.

4. Swinnen B, Robberecht W. The phenotypic variability of amyotrophic lateral sclerosis. Nat Rev Neurol 2014; 10: $661-670$.

5. Spiro AJ. Minipolymyoclonus - a neglected sign in childhood spinal muscular atrophy. Neurology 1970; 20: 1124-1126.

6. Bhat S, Ma W, Kozochonok E, Chokroverty S. Fasciculations masquerading as minipolymyoclonus in bulbospinal muscular atrophy. Ann Indian Acad Neurol 2015; 18: 249-251.

7. Hallett M. Tremor: pathophysiology. Parkinsonism Relat Disord 2014; 20(Suppl. 1): S118-S122.

8. Elble RJ. Physiologic and enhanced physiologic tremor. In: Hallett M, ed. Handbook of Clinical Neurophysiology. New York, NY: Elsevier Science BV, 2003: 357-364.

9. Deuschl G, Raethjen J, Lindemann M, Krack P. The pathophysiology of tremor. Muscle Nerve 2001; 24: 716735.

10. Mehta AR, Brittain JS, Brown P. The selective influence of rhythmic cortical versus cerebellar transcranial stimulation on human physiological tremor. J Neurosci 2014; 34: 7501-7508.
11. Raethjen J, Deuschl G. The oscillating central network of Essential tremor. Clin Neurophysiol 2012; 123: 61-64.

12. Gerwig M, Kolb FP, Timmann D. The involvement of the human cerebellum in eyeblink conditioning. Cerebellum 2007; 6: 38-57.

13. Farmer SF, Bremner FD, Halliday DM, Rosenberg JR, Stephens JA. The frequency content of common synaptic inputs to motoneurones studied during voluntary isometric contraction in man. J Physiol 1993; 470: 127-155.

14. Halliday DM, Rosenberg JR, Amjad AM, Breeze P, Conway BA, Farmer SF. A framework for the analysis of mixed time series/point process data - theory and application to the study of physiological tremor, single motor unit discharges and electromyograms. Prog Biophys Mol Biol 1995; 64: 237-278.

15. Antelmi E, Di Stasio F, Rocchi L, et al. Impaired eye blink classical conditioning distinguishes dystonic patients with and without tremor. Parkinsonism Relat Disord 2016; 31: 23-27.

16. Homberg V, Hefter H, Reiners K, Freund HJ. Differential effects of changes in mechanical limb properties on physiological and pathological tremor. J Neurol Neurosurg Psychiatry 1987; 50: 568-579.

17. Rothwell JC. Physiology and anatomy of possible oscillators in the central nervous system. Mov Disord 1998; 13(Suppl. 3): 24-28.

18. Prochazka ATP. Instability in human forearm movement studied with feed-back-controlled muscle vibration. J Physiol 1998; 402: 421-442.

19. Britton TC, Thompson PD, Day BL, Rothwell JC, Findley LJ, Marsden CD. Modulation of postural tremors at the wrist by supramaximal electrical median nerve shocks in essential tremor, Parkinson's disease and normal subjects mimicking tremor. J Neurol Neurosurg Psychiatry 1993; 56: 1085-1089.

20. Elble RJ, Higgins C, Hughes L. Phase resetting and frequency entrainment of essential tremor. Exp Neurol 1992; 116: 355-361.

21. Helmich RC. The distributed somatotopy of tremor: a window into the motor system. Exp Neurol 2013; 241: $156-158$.

22. Stein RB, Oguztoreli MN. Tremor and other oscillations in neuromuscular systems. Biol Cybern 1976; 22: 147157.

23. Hoffland BS, Kassavetis P, Bologna M, et al. Cerebellum-dependent associative learning deficits in primary dystonia are normalized by rTMS and practice. Eur $J$ Neurosci 2013; 38: 2166-2171.

24. Kronenbuerger M, Gerwig M, Brol B, Block F, Timmann D. Eyeblink conditioning is impaired in subjects with essential tremor. Brain 2007; 130(Pt 6): 1538-1551.

25. Keil C, Prell T, Peschel T, Hartung V, Dengler R, Grosskreutz J. Longitudinal diffusion tensor imaging in amyotrophic lateral sclerosis. BMC Neurosci 2012; 13: 141.

26. Schoenfeld MA, Tempelmann C, Gaul C, et al. Functional motor compensation in amyotrophic lateral sclerosis. J Neurol 2005; 252: 944-952.

27. Tan RH, Kril JJ, McGinley C, et al. Cerebellar neuronal loss in amyotrophic lateral sclerosis cases with ATXN2 intermediate repeat expansions. Ann Neurol 2016; 79: 295-305.

28. Nakano T, Nakaso K, Nakashima K, Ohama E. Expression of ubiquitin-binding protein p62 in ubiquitin-immunoreactive intraneuronal inclusions in 
amyotrophic lateral sclerosis with dementia: analysis of five autopsy cases with broad clinicopathological spectrum. Acta Neuropathol 2004; 107: 359-364.

29. Takahashi H, Oyanagi K, Ikuta F, Tanaka M, Yuasa T, Miyatake T. Widespread multiple system degeneration in a patient with familial amyotrophic lateral sclerosis. $J$ Neurol Sci 1993; 120: 15-21.

30. Renton AE, Majounie E, Waite A, et al. A hexanucleotide repeat expansion in C9ORF72 is the cause of chromosome 9p21-linked ALS-FTD. Neuron 2011; 72: 257-268

31. Troakes C, Maekawa S, Wijesekera L, et al. An MND/ ALS phenotype associated with $\mathrm{C} 9$ orf72 repeat expansion: abundant p62-positive, TDP-43-negative inclusions in cerebral cortex, hippocampus and cerebellum but without associated cognitive decline. Neuropathology 2012; 32: 505-514.

32. Gellera C, Ticozzi N, Pensato V, et al. ATAXIN2 CAG-repeat length in Italian patients with amyotrophic lateral sclerosis: risk factor or variant phenotype? Implication for genetic testing and counseling. Neurobiol Aging 2012; 33: 1847.e15-21.

33. Afshar P, Ashtari N, Jiao X, et al. Overexpression of human SOD1 leads to discrete defects in the cerebellar architecture in the mouse. Front Neuroanat 2017; 11: 22.

34. Vance C, Rogelj B, Hortobagyi T, et al. Mutations in FUS, an RNA processing protein, cause familial amyotrophic lateral sclerosis type 6. Science 2009; 323: 1208-1211.

35. Rademakers R, Stewart H, Dejesus-Hernandez M, et al. Fus gene mutations in familial and sporadic amyotrophic lateral sclerosis. Muscle Nerve 2010; 42: 170-176.
36. Huang EJ, Zhang J, Geser F, et al. Extensive FUSimmunoreactive pathology in juvenile amyotrophic lateral sclerosis with basophilic inclusions. Brain Pathol 2010; 20: 1069-1076.

37. Wu YR, Foo JN, Tan LC, et al. Identification of a novel risk variant in the FUS gene in essential tremor. Neurology 2013; 81: 541-544.

38. Zheng W, Deng X, Liang H, et al. Genetic analysis of the fused in sarcoma gene in Chinese Han patients with essential tremor. Neurobiol Aging 2013; 34: 2078e3-4.

39. Rajput A, Rajput AH, Rajput ML, et al. Identification of FUS p. R377W in essential tremor. Eur $J$ Neurol 2014; 21: 361-363.

40. Merner ND, Girard SL, Catoire H, et al. Exome sequencing identifies FUS mutations as a cause of essential tremor. Am J Hum Genet 2012; 91: 313-319.

41. Nishimura AL, Mitne-Neto M, Silva HC, et al. A mutation in the vesicle-trafficking protein VAPB causes lateonset spinal muscular atrophy and amyotrophic lateral sclerosis. Am J Hum Genet 2004; 75: 822-831.

42. Di L, Chen H, Da Y, Wang S, Shen XM. Atypical familial amyotrophic lateral sclerosis with initial symptoms of pain or tremor in a Chinese family harboring VAPB-P56S mutation. J Neurol 2016; 263: $263-$ 268.

43. Deuschl G, Bain P, Brin M. Consensus statement of the movement disorder society on tremor. Ad Hoc Scientific Committee. Mov Disord 1998; 13(Suppl. 3): 2-23.

44. Brennan KC, Jurewicz EC, Ford B, Pullman SL, Louis ED. Is essential tremor predominantly a kinetic or a postural tremor? A clinical and electrophysiological study. Mov Disord 2002; 17: 313-316. 